\title{
Eight
}

\section{ATHEISTIC ANTHROPIC COSMOLOGY}

We live in a remarkable universe. Among possible universes, the fact that our universe is compatible with and supports our existence makes it extraordinary. Any universe in which intelligent creatures like ourselves could exist would be a fabulous universe, for lifeless universes could be produced in an infinite number of ways, but only a few highly contrived ways can produce life-sustaining ones. Some cosmologists claim that there is only one way to make a lifesustaining universe. ${ }^{\prime}$

Except for the Greek and Roman Atomists, most pre-modern thinkers assumed that some kind of special relationship exists between humanity and the universe, that humankind is made for the universe and the universe for humankind. Teleology means purposiveness. Western philosophers and theologians traditionally believed that we live in a purposeful universe, that teleology is an important and conspicuous feature of nature.

Because seventeenth and eighteenth century mechanistic materialists vigorously attacked cosmic teleology, non-teleology became a fundamental presumption of modern science. To exorcize Aristotelian final causes and all other purposes from nature, modern natural science aspires to explain everything in terms of formal causes (natural laws), efficient causes (energy transfers) and material causes (spatially extended entities). Naturalism, as explained in Chapter Two, made anti-teleology a fundamental metaphysical principle. Humanistic Naturalists think that we and similar organisms have purposes, but not the whole of nature, and not some purely fanciful supernatural ground or cause of nature.

Teleology on a small scale inescapably reappears in natural and social sciences like biology and psychology, despite the domination of scientific orthodoxy by naturalistic metaphysics. Teleology also resurfaces on a larger scale in recent cosmology as the Anthropic Principle, so named by the physicist Brandon Carter in 1974. Carter was not the first to notice that the universe is fine-tuned for the emergence of human life, but he first christened this the "Anthropic Principle."

\section{The Anthropic Principle and Cosmic Purpose Without God}

Cosmologists are again finding purpose in the universe, but we should not jump to the wrong conclusion. Most of them do not wish to revitalize and embrace a new version of the religious Argument from Design for the existence of God. In fact, most Anthropic Cosmologists are thoroughly atheistic and naturalistic and aspire to show how there can be cosmic teleology without God. Most of them make two fundamental claims: We live in a purposive universe that is 
exquisitely fine-tuned for the production of intelligent life, and God is not required to explain such conspicuous cosmic purposiveness.

In their 1988 book, The Anthropic Cosmological Principle, John D. Barrow and Frank J. Tipler thoroughly develop Anthropic Cosmology, explore its history, and defend it against many challenges. ${ }^{3}$ Additional prominent contributors to the development of Anthropic Cosmology include Brandon Carter, P. C. W. Davies, ${ }^{4}$ Robert H. Dicke, ${ }^{5}$ Freeman Dyson, ${ }^{6}$ Stephen Hawking, ${ }^{7}$ B. J. Carr and Martin Rees, ${ }^{8}$ John Leslie, ${ }^{9}$ and John A. Wheeler. ${ }^{10}$

Anthropic Cosmologists do not want to overturn the Copernican revolution and reposition mankind in some privileged position in the center of the universe. They believe that we can have a special place in the universe without being at its physical center. As Barrow and Tipler express it, "Although we do not regard our position in the Universe to be central or special in every way, this does not mean that it cannot be special in any way." Brandon Carter remarks that "Our location in the Universe is necessarily privileged to the extent of being compatible with our existence as observers."12

The purpose of the universe, says Anthropic Cosmology, is to produce complex intelligent forms of life, like human life. The word "anthropic" is a bit misleading, suggesting that we human beings are the only complex, intelligent, living things in the universe, that the universe is designed to produce only us; but these intimations are not really intended. This would distance Anthropic Cosmology too far from what many astrophysicists misleadingly call "the Copernican revolution." Copernicus himself did not doubt that God created the universe for mankind, even if our earth orbits the sun rather than vice versa.

Anthropic Cosmologists agree that other complex intelligent life forms may exist on planets in other solar systems. After all, a hundred and fifty billion stars exist in our Milky Way; at least a hundred twenty-five billion other galaxies of equal or greater complexity exist in the observable universe; and intelligent life is very likely to exist elsewhere. The basic chemistry for life is widespread. At the moment, neither the existence of life outside our solar system nor the degree of its prevalence elsewhere have been confirmed; but many planets orbiting other suns have now been located, and many cosmologists are convinced that life is prevalent throughout the universe..$^{13}$

Anthropic Cosmology tends to be excessively anthropocentric only in the sense that it exhibits a definite bias toward intelligence. It assumes that other forms of life less intelligent than ourselves have little if any intrinsic worth, and that intelligence as a value epitomizes even if it doesn't exhaust our own worth. To avoid these errors, a broader Biopic Cosmology is needed, one that recognizes the great intrinsic worth of an immense variety of terrestrial and possible extraterrestrial forms of life, one that is not biased against the non-cognitive dimensions of human and non-human nature. Human life stands at the apex of complex, intelligent, affective, volitional life on earth. Yet, we differ from nonhuman animals only in degree, not in kind. Degrees of intelligence and many 
other traits that make life worth living for its own sake are shared with other terrestrial animals.

According to the Biopic Cosmology advanced in this book, the purpose of the universe is to produce "an immense variety of forms of experience, love, loyalty, enjoyment, responsibility, initiative, creativity, achievement, and satisfaction, even at the price of conflict." ${ }^{14}$ These desirable traits enrich and exist only in the lives of concrete conscious individuals, the proper locus of intrinsic worth. Valuable individual lives need be neither anthropic, that is, humanoid, nor carbon-based; but carbon, hydrogen, oxygen, and nitrogen have properties that greatly favor the origin and evolution of life. ${ }^{15}$ These elements exist abundantly throughout the universe. For all we know, the purpose of the universe may be fulfilled only on our earth; but this seems unlikely.

Spectroscopic analysis discloses that the basic chemistry of the universe is the same throughout; and elements and compounds necessary for the formation of carbon-based lives are widely distributed throughout the universe. Carbon-based living things require hydrogen, oxygen, nitrogen, phosphorus, and traces of many other elements; but these too are found in ample quantities sufficient for life throughout our galaxy and in myriads of others. Many other special conditions may be necessary for life, so we are admittedly uncertain about the prevalence of life throughout the cosmos. Non-carbonaceous lifeforms are possible, even if carbon specially favors life. Complex non-carbonaceous life-forms are improbable; their existence has not yet been confirmed; but if any exist, the universe is even more suitable for the production of complex and valuable conscious living things than we commonly suspect.

Considering mainly carbon-based life, our universe manifests a huge number of "extraordinarily finely tuned coincidences,"16 as Barrow and Tipler put it, that seem designed intentionally to create life as we know it. More details of this fine-tuning for life will be given in the next chapter; but we must first examine several meanings of the Anthropic Principle and note that most Anthropic Cosmologists favor only those meanings that exclude Divine foresight, planning, and purpose.

Barrow and Tipler say that the Anthropic Principle has at least three meanings, the first two of which were recognized by Brandon Carter: (1) The Weak Anthropic Principle says nothing more than that we would not be here unless the universe were compatible with our existence. (2) The Strong Anthropic Principle affirms that the universe must produce human or intelligent existence. This "must" generates Theistic, Quantum Observership, and Infinite World-Ensemble (Big Fizz and Big Divide) interpretations. (3) The Final Anthropic Principle says that we exist for the sake of a final Omega Point. Each version has its weaknesses, and the whole enterprise of Anthropic Cosmology is highly controversial. Atheistic Anthropic Cosmologists accept either the Weak Principle, the second or third interpretations of the Strong Principle, or the Final Principle, according to which God's non-existence is only temporary. 
They generally reject the theistic interpretation of the Strong Principle that is defended in this and following chapters.

After the Weak Principle is given a metaphysical underpinning, it is indistinguishable from the world-ensemble interpretation of the Strong Principle. Because it adds nothing to it and subtracts nothing from it, the fatal flaws of the former are also ruinous to the latter. Only the Weak World-Ensemble Anthropic Principle, the Strong Quantum Observership Principle, and the Final Anthropic Principle are viable options for Atheistic Anthropic Cosmology; but do they hold up under critical analysis?

\section{The Weak and Strong Anthropic Principles}

The Weak Anthropic Principle affirms nothing more than that we would not be here if the universe were not compatible with and supportive of our existence. ${ }^{17}$ Brandon Carter's formulation of the Weak Anthropic Principle says that "What we can expect to observe must be restricted by the conditions necessary for our presence as observers."18 As Stephen Hawking put it, "We see the universe the way it is because we exist." tautology, though it is occasionally denounced as such. Nevertheless, it is singularly uninformative. It tells us nothing more than that we are here only because the universe is compatible with and supports our existence. It gives no reasons why.

In its purest form, the Weak Anthropic Principle offers no reason for the compatibility between the universe and ourselves. Anthropic Cosmology becomes interesting and informative only when someone tries to explain why we live in a universe that is compatible with and supportive of our existence. Part of the answer is very obvious: if the universe were otherwise, we would not be here asking the question, and Anthropic Cosmologists would not be here concocting the answers. This is so patently obvious and unilluminating that Weak Anthropic Cosmologists usually take further steps. They advance from Weak to Strong. They offer a metaphysical underpinning for the Weak Anthropic Principle-an infinite worlds metaphysics; but this converts the Weak Anthropic Principle into an infinite universe interpretation of the Strong Anthropic Principle. These two options, having become one, will shortly be evaluated together.

As Brandon Carter formulated it, the Strong Anthropic Principle says that "The Universe must be such as to admit the creation of observers within it at some stage." ${ }^{20}$ The emphasis here is on the word "must," but what Carter meant by this is unclear. It suggests that no universe can come into being that lacks intelligent observers altogether, that some observational selection principle excludes universes inhospitable to our kind of life. What could this selection principle be? Barrow and Tipler consider three possibilities. ${ }^{21}$

First, the universe may have been designed deliberately by Divinity, by some "Supercalculating Intellect," as Fred Hoyle expressed it, who intended to 
create and sustain finite, conscious, intelligent beings. Atheistic Anthropic Cosmologists like Barrow and Tipler reject this option in favor of the second or third. They are determined to give us teleology without Theism-at least until Omega comes in all its glory.

Second, observers may be necessary to bring the world into being, as claimed by Niels Bohr's Copenhagen interpretation of quantum mechanics and John A. Wheeler's theory of Quantum Observership (discussed and refuted already), and by the Participatory Anthropic Principle.

Third, infinitely many worlds co-existing in Superspacetime would necessitate our existence because they actualize all possibilities. Worlds containing intelligent life are possible worlds, so a few such worlds will be actual if all possibilities are actualized somewhere. We just happen to live in one of these. Some gloves will fit given an infinite number of gloves. No Supercosmic Intelligence is required to explain why we live in a universe in which astonishing cosmic coincidences conspire to produce and support our existence. The Principle of Plenitude insures the existence of infinitely many worlds; supposedly it explains everything, although it really explains nothing. This atheistic infinitely many worlds metaphysics must now be examined carefully. After finding it wanting, the Final Anthropic Principle will be examined and dispose of in the next chapter. Theistic options will fill the concluding chapters

Atheistic versions of the Strong Anthropic Principle usually appeal to the existence of infinitely many worlds to explain why, without God, we live a universe that is exquisitely designed to support conscious, intelligent, sensitive life. Given an infinite number of possible universes, most of which are doubtless incompatible with life, why do we live in one that supports life? According to the metaphysical Principle of Plenitude, all possible universes, an infinite number of them, must actually exist. Possibility is identical with actuality. Given an infinite number of diverse universes, at least a few of them will support life accidentally; and we just happen by chance to be in one that does. No God planned it. The shoe fits; but if an infinite number of different shoes exist, at least one is bound to fit. This is almost self-evident; but it is false!

At least four infinite universe cosmologies would serve the metaphysical purposes of Atheistic Anthropic Cosmology. First, as in Plasma Cosmology, a single universe may be infinite in space and time and contain an infinite number of relatively isolated metagalaxies, most of which are hostile to life, but a few will be randomly life-supporting. We just happen by chance to live in a supportive metagalaxy.

Relatively isolated metagalaxies belong to a single spatiotemporal universe presumably because they continue to have causal contact with other metagalaxies along their edges; but these edges may be so far removed from particular observers like us that we cannot detect them. The relative isolation of metagalaxies cannot be complete because, if complete, this option is indistinguishable from Big Fizz or Big Divide world-ensemble cosmology. John Leslie 
points out that many worlds theorists disagree about whether many worlds interact causally. ${ }^{22}$ If worlds do interact causally in Big Fizz or Big Divide theories, they no longer differ from positions that affirm relatively but not completely isolated metagalaxies in a single universe. This is the old "same universe" quandary all over again.

Second, as in Oscillation Cosmology, an infinite succession of consecutive universes with different laws and initial conditions might exist, being separated temporally rather than spatially from one another. In most cosmic epochs, laws and conditions would be hostile to life; but within an infinite number of diversified tries, a cosmic epoch will occasionally come along that supports life. Periodically, in an infinite number of diversified successive universes, one will be suitable for habitation by conscious, intelligent, living beings like us. We just happen by accident to live in such a one. No observers, astronomers, philosophers, or ordinary people inhabit most of the others.

Third, in Big Fizz world-ensemble cosmology, an infinite number of spatially co-existing universes with different laws and initial conditions are promiscuously spawned by Mother Spacetime. Some of these may then oscillate, so this metaphysics may be combined with the preceding. Infinitely many co-existing worlds are completely separated from and have no causal contact with one another in infinite Superspace. Given an infinite number of structurally diverse contemporary universes, most will be incompatible with life; but a few will support life. By chance, we just happen to exist in one of these. In most of the others, no observers, no scientists, and no inquirers wonder about the purpose of the universe.

Fourth, in Big Divide many worlds cosmology, every universe branches profusely and indiscriminately into new and otherwise causally isolated parallel universes at every turn of events. All possibilities for every reality are actualized, and it takes an infinite number of universes to make it all happen. When parallel universes face a choice between life and no life, they divide; and at least one universe containing life is created. Given an infinite number of branches, some will be life-supporting. By pure chance we live in a life-supporting offshoot. Our own universe is constantly sprouting new universes that actualize every possibility open to every point of space and every instant of time, but most universes are uninhabited.

By appealing to one or more versions of infinite worlds metaphysics, Atheistic Anthropic Cosmology tries to account for the life-supporting purposiveness of our universe without resorting to an intelligent and purposive God. It offers teleology without theology, a universe fine-tuned for life purely by accident. But which is easier to swallow, an unseen transcendent infinite God, or an unseen transcendent infinity of worlds? Should an intelligent person affirm infinitely many worlds that don't know what they are doing, or an infinite God who knows what he is doing? The following considerations should facilitate a more informed decision. 


\section{Critique of Infinite World-Ensemble Teleology}

\section{A. Non-Empirical Status}

Atheistic interpretations of the Weak and Strong Anthropic Principles do not explain purpose in the universe until they trot out an infinite worlds metaphysics. Unfortunately, we have no direct experiential or legitimate inferential access to even one other world, much less an infinite number of them. Given an infinite number of universes, Atheistic Anthropic Cosmologists claim, there can be cosmic purpose without God; but there is no good reason to give this, whether it be an infinite number of distant metagalaxies, antecedent universes, disconnected worlds co-existing in Superspacetime, or worlds branching from our existing universe. If quantum theory rejects as empirically meaningless the objective existence of unobservable quantum states, it should also refuse to proliferate unobserved and unobservable quantum and non-quantum worlds $a d$ infinitum.

Barrow and Tipler acknowledge the non-empirical status of an infinite number of metagalaxies and oscillating universes, but they seem blind to the non-empirical status of the infinite world-ensemble (Big Fizz) option that they embrace. Eric Lerner's Plasma Cosmology postulates an infinity of loosely connected metagalaxies. Barrow and Tipler find the same postulate in the publications of G. F. R. Ellis. ${ }^{23} \mathrm{After}$ briefly explaining his position, Barrow and Tipler say that "It is hard to evaluate this idea any further, but one thing is certain: if it is true then it is certainly not original. ${ }^{24}$ The theory is indeed hard to evaluate because it is nothing more than sheer fantasy!

Barrow and Tipler are much clearer about the non-empirical status of Oscillation Cosmology, remarking that "It is far from being testable." ${ }^{25}$ They fail somehow to see that this is true also of their own infinite world-ensemble metaphysics. If any version of an infinite worlds metaphysics is true, we merely human mortals could never know it; but we have no good reasons for thinking that it is true.

Oscillation Cosmology is not testable, according to Barrow and Tipler. Inconsistently, they later suggest that it actually makes a testable prediction, one that they are unwilling to accept. Given an infinite number of oscillations, if the basic laws and constants of nature change with each bounce,

Sooner or later the geometry would be exchanged for a noncompact structure bound to expand for all future time. The Universe should currently be 'open' destined to expand forever since this state will always be reached after a finite series of oscillations. ${ }^{26}$

Barrow and Tipler do not realize that this concession is utterly devastating to Oscillation Cosmology. Every cosmic epoch in an infinite oscillating series is 
preceded by an infinite number of antecedent universes persisting collectively through an infinite past. An infinite amount of time is quite long enough for "sooner or later," so every universe in such a series should be open. For this reason alone, the very idea of an infinite number of antecedent universes is completely untenable.

Our universe's being open or closed is testable, in principle capable of being decided. All we have to do is find out how much mass/energy the entire universe contains. Unfortunately, this is no easy task; but, given what we now know, as explained earlier, the available evidence strongly indicates that we live in an open universe. Because of their theoretical biases, Barrow and Tipler opt for a closed universe. They recognize that an infinite series of oscillating diversified universes eventually achieves a geometry that destines a terminal member to expand forever; but they still insist that our universe is closed in order to secure their Final Anthropic Principle; only a closed universe can achieve what they think is its ultimate purpose-the Omega Point.

A closed universe is implausible indeed if grounding the Final Anthropic Principle is the best reason that can be given for it, but more about that in the next chapter.

\section{B. The Principle of Plenitude}

Weak and Strong World-ensemble Anthropic Principles presuppose the validity of the Principle of Plenitude, or the Principle of Fecundity, as Robert Nozick calls it, ${ }^{27}$ which says that all possible worlds are actual worlds. Here is the heart of the Atheistic Anthropic position on the cause of our life-producing Big Bang. The existence of our universe is required by the Principle of Plenitude-an abstract, non-empirical, disembodied, supercosmic normative principle which necessitates that all possible worlds must be actual worlds. But what grounds and drives this supercosmic compulsion to actualize all possibilities? What gives it the power to actualize its ideal? Atheistic Anthropic Cosmologists have no clear answer except that it is not Divine, at least not yet!

The ancestry of the Principle of Plenitude is unquestionably theological. Plenitude is definitely not an empirical principle or a verified discovery of empirical natural science. Arthur Lovejoy, who coined the phrase, classified it as "metaphysical theology."28 Lovejoy traced the principle back to Plato's conviction that the actual world copies every one of, and every possible combination of, the eternal forms because the richer reality is, the better or more perfect it is, and because the Divine Demiurge would not be good, perfect, complete, and divine unless he actually is or creates everything that he possibly could be or create. ${ }^{29}$

As Lovejoy interpreted it, the Platonic Principle of Plenitude requires the existence not only of every possible grade or kind of being, but also of every possible individual at every level, since the forms combine to constitute individ- 
uals. Plenitudists have wavered on the issue of whether plenitude requires merely that all possible kinds of things be actual, or whether all possible individuals of every kind are also required.

The Greek Atomists hypothesized the existence of infinitely many worlds within the infinite void of space, but Plato and the Medieval theologians who followed him did not derive infinitely many distinct universes from the Principle of Plenitude. They maintained that for both God and the creation to be perfect, God only had to create representatives of all possible compatible kinds, degrees, or levels of being within this one world. So how many continuous grades of being are there? An infinite number, says Lovejoy, though he admits that neither the Greek philosophers who espoused plenitude nor the Medieval theologians who embedded it into Christian theology were always fully aware of its implications; they often combined it with incompatible qualifications.

The levels of being that the Medieval theologians actually identified were very finite; and they, like Plato, subscribed to incompatible premises that prevented infinite plenitude from really adding up to infinity. God in absolute selfsufficiency needs nothing, not even to create, they held; and God's being and goodness are in no way enriched by creation. God creates only what He freely chooses to create, even though Plenitude requires Him to create absolutely everything. Further, a morally good God creates only a morally good world, even though plenitude necessitates the creation of every possible world, no matter how nasty. The good world that God created contains only those kinds of being and those individual beings that are at least roughly harmonious with one another on the whole, but plenitude requires that every possibility be actual, whether harmonious or discordant, whether good or bad.

Traditional Christian theologians through the centuries so restricted the Principle of Plenitude that it did not readily translate into infinite creation. Only God is actually infinite, they held; the created universe, though in some vague sense complete and perfectly ordered from the beginning, is finite. God's plenitude is in himself-an absolute plenitude of being in which there are no unactualized possibilities; but Divine plenitude does not entail creating a truly infinite world or an infinite number of worlds. In himself, quite apart from any and all creation, God is pure being, everything that a perfect being could possibly be, they thought; but God creates only what he wills to create; and he willed to create a finite world which, in the light of modern cosmological knowledge, seems rather paltry.

Before Copernicus and the dawning of modern cosmology, the Christian theologians who espoused plenitude believed that God created a multi-storied finite universe with the earth at is center, surrounded by a finite number of concentric celestial spheres that separate us from God's Heaven. In the closest celestial sphere, a finite number of "wandering stars"-the planets, understood by many to be embodied angels-rotated around the earth. Our sun and moon were classified as planets. In the outermost spheres were the "fixed stars"- 
those we now know to have relatively fixed positions in our Milky Way. Many Medieval theologians thought the fixed stars to be either embodied angels or moved by angels who exist on a higher link in the chain of being. All heavenly bodies were mistakenly thought to rotate around the earth in perfect circular orbits. Non-circles are imperfect, and God created nothing imperfect. God sets the outermost celestial sphere into motion, and everything between there and the earth is moved by other motions in the next highest sphere. Despite plenitude, also affirmed, this great chain of being manifests no infinite regress (thus, God exists) and no infinite continuum of grades of being.

From the beginning of Christian cosmology, and through the Middle Ages, the Principle of Plenitude applied most conspicuously to God in himself; it really did not apply to the created world-even when it did! Christian theologians primarily emphasized the infinite plenitude of Divine Being, not the infinite plenitude of creation. Plato thought that the supreme form of The Good required that all the forms be actualized or copied within creation; and his creative and beneficent Demiurge executed this requirement.

For St. Thomas Aquinas, God in himself is pure being, pure actuality in whom there are no unactualized divine possibilities; but this did not translate into infinitely many created universes, partly because no created things made any internal difference to (had any real or internal relations with) God, and partly because a good God would create only a good world, not all possible evil worlds. Aquinas considered the hypothesis that God created infinitely many worlds because his infinite power ${ }^{30}$ or goodness ${ }^{31}$ seems to require infinite creativity, but he rejected it for reasons not always very clear or defensible. Still, something that superficially resembles infinite plenitude of creation does appear in Aquinas. He held that the perfection of the finite world requires God to create representatives of innumerably many species or grades of created beings; but Aquinas never says all or infinitely many species. Many things that might exist do not, he insisted, and only those species were created that harmonize on the whole with the existence of other species, especially humankind. ${ }^{32}$

Still, we might wonder, as did Lovejoy, why an infinite God, who is everything that he could be, did not create everything that he could create, and why infinitely many degrees of created beings could not exist within one infinite universe. Why and how could God's creativity be limited when God himself is pure, infinite, limitless, actual being in whom there are no unactualized potentialities at all?

By affirming repeatedly that God wills to create only that which the divine intellect proposes under the form of goodness, Classical Theologians like St. Thomas Aquinas easily avoided the difficulty that some possible worlds would be so horrible that a morally virtuous God would never create them. The perfection of unqualified plenitude of creation is clearly incompatible with the perfection of moral goodness or righteousness. A benevolent God would not create all possible worlds. The problem of theodicy, addressed in more depth in Chapter 
Eleven, says that whether a good and intelligent God could have created this world of woe is seriously doubtful; but much worse worlds are possible, thus actual somewhere, under the unqualified aegis of the Principle of Plenitude.

Historically, the Principle of Plenitude did not originate with empirical natural science. It sprang from a very peculiar but highly influential ancient Greek way of conceiving of divine perfection. Amazingly, despite its lack of empirical origin or justification, the principle is alive and well today among scientific-minded cosmologists. To avoid God, many contemporary Anthropic Cosmologists affirm either a single infinite spatiotemporal universe or an infinite number of universes in time and/or space. Just what differentiates them depends upon how universes are distinguished, as articulated earlier.

The infinite worlds metaphysics of today's Weak and Strong Anthropic Principles is nothing but metaphysical theology without God; yet, without God the whole rationale for it is lost. Unless Plato's ideal of divine perfection is first accepted, we have no good reason to affirm any version of the Principle of Plenitude or the infinity of this or any other universes. Also, many theists have very different concepts or ideals of divine perfection; and Chapter Ten will show how divine perfection may be conceived without Plenitude.

Today's world-ensemble cosmologists presuppose that the disembodied Principle of Plenitude is the ultimate, final, and efficient cause of our lifeproducing Big Bang, just as it is the ultimate cause of every other universe, an infinite number of them. What caused the Big Bang? The Principle of Plenitude!

Yet, we have no good reasons to believe that disembodied, abstract principles are anything more than impotent Aristotelian formal or final causes. The main difference between Aristotelian and Platonic approaches to metaphysics is that Platonists think that universals, including abstract principles, can and do exist and exercise efficient causation without being located or embodied in actual entities. By contrast, Aristotelians are persuaded that universals exist only in actual individuals and that abstract principles can be causally effective only through embodied conscious individuals who understand and act upon them. On this issue, experience always favors the Aristotelian approach. Experience never supports Platonism. That is quite enough to make the Aristotelian view, but not the Platonic, rationally warranted.

In his brilliant book, Universes, John Leslie gives a highly persuasive Anthropic Argument from Design for the existence of God; but by "God" Leslie does not mean an actual personal conscious mind who understands and acts knowingly and deliberately upon abstract principles. Instead, he defines "God" as nothing more than an abstract Neoplatonic principle. For him, God is

the creatively effective ethical requirement that there be a good universe or universes. Or again he is the Principle that the ethical need for a universe or universes is itself responsible for the actual existence of that universe or those universes. ${ }^{33}$ 
But who or what has this need? Nothing; no one!

If "there is," as Leslie says, an ethical need for infinitely many universes, his Neoplatonic God is nothing but the abstract Platonic Principle of Plenitude, except that unrestricted plenitude entails both bad and good universes, since it supposedly produces all possible universes. In response to the objection that his theology provides no mechanism by which ethical needs produce their effects, Leslie explains that "Neoplatonism is the view that ethical needs are themselves creatively effective, unaided by any mechanism." 34

No Aristotelian or Whiteheadian metaphysician would be convinced! Alfred North Whitehead's "Ontological Principle" says that

The reasons for things are always to be found in the composite nature of definite actual entities-in the nature of God for reasons of the highest absoluteness, and in the nature of definite temporal actual entities for reasons which refer to a particular environment. ${ }^{35}$

If Aristotle and Whitehead are right, disembodied normative principles cannot be efficient causes of anything, much less of universes. Only definite actual entities like human beings or an embodied personal God can understand and act knowingly on normative principles to make them efficacious. Principles themselves are only formal causes; they cannot act by themselves. Abstract principles have no needs at all, especially no ethical needs for life-supporting universes. This Aristotelian/Whiteheadian position is universally confirmed by experience. This is really all that needs to be said for it-and against Platonism.

M. A. Corey protests that Process Theists cannot affirm that God unilaterally determined the initial conditions of the universe so that they would be suitable for the later evolution of complex forms of life because these initial conditions themselves would have been free to resist the divine will, and some would have done so. ${ }^{36}$ This spurious objection also treats abstractions-the laws of physics, the constants of nature, and the aggregate quantity of initially undifferentiated mass/energy in the nascent universe-as if they were concrete individuals capable of making choices; but no process thinker would so regard them. In fact, since the reasons for things must always be traced back to individual entities, and since God is the only individual entity capable of determining the initial conditions that govern and limit all lesser individual entities, we could and should expect God to set the initial limits for all creation. Disembodied, unindividuated abstractions can neither resist the will of God nor actualize any possibilities, much less all of them. Impersonal aggregates like door knobs and just-created, pure, undifferentiated, grandly-unified energy have no consciousness or freedom to resist God's will, just as impersonal principles have no power to accomplish anything by themselves. Ethical needs for good universes, or for all possible universes, must exist in some actual entity, if they exist at all; but these needs are not compatible with one another. 


\section{Infinity $=$ All Possibilities}

Let us grant for the sake of the argument that the Principle of Plenitude might be the efficient cause of whole universes, either temporally as an infinite linear series of oscillating worlds, or spatially as an infinite number of co-existing worlds, or both. Fully deployed, this principle demands the existence of an infinite world or number of worlds in which every possible grade of reality, every possible individual entity, and all possible qualities and relations are actualized. In physical terms, this principle necessitates the realization of every possible quantity, quality, kind, and combination of mass/energy. Each tiny variation calls for a whole new universe in which everything else in the universe has a new relationship with that variation, no matter how minute. Oscillation Cosmologies express the temporal alternative; and co-existing universes in Big Fizz and Big Divide Cosmologies express the spatial option, which may be combined with the temporal.

Logically, a single temporal series of oscillating universes can never absolutely fulfill Plenitude of Creation, for an infinite number of spatially coexisting universes are also possible; but neither can Mother Spacetime's Big Fizz, for possibly only a single temporal strand of oscillating universes exists. Many possible worlds and combinations of them exclude other possible worlds and combinations. Absolute plenitude of creation is utterly unintelligible!

We have no good reasons to believe in any other worlds, much less infinitely many of them. More seriously, even if infinitely many universes exist, infinity as such does not explain why we live in a life-supporting universe, despite what most atheistic cosmologists assume. Why must an infinitely prolonged spatial or temporal series of worlds actualize all possibilities, or even all logically compatible possibilities? Plato's God and the God of classical western theology supposedly, but not always consistently, actualized all possibilities knowingly and deliberately; but would infinity alone do so in the absence of Divine Agency, as Atheistic Anthropic Cosmologists contend? No, for infinite numbers alone do not necessitate or entail endless categorial, individual, qualitative, and relational diversity, despite what most atheistic philosophers and astrophysicists think. We must take a careful look at this last great unchallenged dogma of unwarranted metaphysics.

By assuming finite matter enduring for infinite time, David Hume tried to escape the theological implications of the Argument from Design by arguing:

Instead of supposing matter infinite, as Epicurus did; let us suppose it finite. A finite number of particles is only susceptible to finite transpositions: and it must happen, in an eternal duration, that every possible order or position must be tried an infinite number of times. Innumerable revolutions produce at last some forms, whose parts and organs are so adjusted as to support the forms amidst a continued succession of matter. ${ }^{37}$ 
What insures that Hume's "innumerable revolutions" will all be different, instead of just being endless repetitions of lifeless sameness? Finite matter existing for infinite time cannot by itself insure infinite diversity. A universe composed of a single hydrogen atom enduring from everlasting to everlasting would fulfill Hume's suppositions. It is a logically possible world, but clearly it would never produce any diversity at all. Neither would a universe of two hydrogen atoms, or three, or four, or many more. All these are logically possible Humean universes. At exactly what point would the addition of finite materiality result in the actualization of infinite diversity? None!

Suppose that infinite quantities of matter exists for infinite time and/or throughout infinite Superspace. Would this insure infinite diversity? Would it necessitate that every possible world, relationship, quality, and individual be actualized? For some theologians, God intentionally makes it so, as Lovejoy points out; Divine perfection deliberately diversifies universes and thereby insures the existence of every good kind and degree of being, but not all possible imperfections. Perhaps divine perfection, Pure Being for and in whom no unactualized possibilities exist, deliberately diversifies universes and guarantees an infinite variety of actual individuals, qualities, relationships, and degrees of being. Hume's disproof of the existence of a purposive God tacitly appeals to a metaphysical principle that requires God, who can supply diversity when sheer numbers cannot!

For Atheistic Anthropic Cosmology, what insures that every possibility is actualized in infinite time and/or space? No one, including David Hume, really knows that time or space are infinite, but let us grant this groundless assumption for the sake of the argument. Infinite time and/or space alone would not insure that every finite combination of individuals and forms will inevitably be actualized because: $i$. This presumption confuses two very different things-numerical spatiotemporal infinity, with an infinite variety of classes, individuals, qualities, and relationships. ii. It clearly lacks a Principle or Agent of Diversification. iii. It definitely confuses infinite possibilities with all possibilities. iv. It mistakenly assumes that infinity would eventually use up all lifeless universes. v. And the very idea of actualizing all possibilities is logically incoherent.

\section{i. Infinity Is Not Infinite Diversity}

The assumption that all possibilities must be actualized in infinite time or space may confuse different orders of infinity. Georg Cantor discovered different orders of infinity, some of which are richer than others. The members of a denumerably infinite set can be put in one to one correspondence with the set of whole numbers, but a nondenumerably infinite set is so rich that its members exceed the set of whole numbers. According to Cantor's theory of transfinites, spatial or temporal infinities are denumerable sets. However, the set of all possible individuals, qualities, and relations is nondenumerably rich. 
Denumerable sets cannot exhaust or use up nondenumerable sets. Denumerably infinite spacetime cannot actualize a non-denumerable infinity of potential individuals, qualities, and relationships. There are just too many of them; but even if this error is not committed, other more serious difficulties plague infinite worlds metaphysics.

\section{ii. An Agent of Diversification Is Needed}

Most seriously, infinity alone does not insure diversity. In infinite worlds metaphysics, lifeless worlds could simply repeat themselves endlessly; and probabilities insure that they would. An infinity of spacetime alone does not guarantee life, the rich environmental order required for its support, or its harmony and goodness on the whole. Endless accidents, or random spontaneous fluctuations alone can insure the actualization of nothing more than an infinite number of garbage universes. Mere infinity cannot guarantee that any universe would ever be life-supporting. For this, an informed choice must be made between lifeless and life-supporting universes, and between good and bad ones; but nothing in the concept of sheer infinity insures any kind of diversity, goodness, or intelligent selection.

Perhaps many worlds metaphysics does not require an infinite number of universes. Some cosmologists suggest that finite but very large number of universes would be sufficient to make a life-supporting universe just happen occasionally. The trouble is that neither finite nor infinite numbers as such insure any diversity at all; if infinity can't do it, neither can finitude. Universes, finite or infinite in number, can be identically lifeless. This is possible and probable. Neither finite nor infinite numbers provide or insure diversification. For that, some additional cause, an agent who selects for diversity, is required.

Life-supporting universes are infinitely improbable. Atheistic Anthropic Cosmologists regularly concede that for every single way to get a life-supporting universe right, there are infinitely many ways to get it wrong. ${ }^{38}$ Something more than mere spatiotemporal infinity is required if conditions essential for life are to converge in some actual universe. The Teleological Argument for the existence of God, developed in a following chapter, says that to get it right, this informed choice must be made by a Superintelligent Being who comprehends infinite errors and blind alleys and chooses against them.

Alfred North Whitehead recognized the necessity for a Divine Principle or Agent of Limitation or Concretion who picks or selects desirable universes to be actualized from unfathomable numbers of undesirable possible worlds. ${ }^{39}$ Nothing in the mere concept of infinity insures any diversity whatsoever, much less the right kind of diversity needed to support highly complex living beings who can live worthwhile lives. An individuated, actualized, and embodied intelligent principle or agent of qualitative selection and diversification of Divine proportions is necessary to make any numerical set of universes exem- 
plify the right kind of qualitative conditions to support worthwhile and complex forms of life.

Neither David Hume nor today's Atheistic Anthropic Cosmologists provide for an intelligent, or even for an unintelligent, agent of qualitative limitation and diversification. Atheistic Anthropic Cosmologists dismiss the Strong Theistic Anthropic Principle much too quickly.

\section{iii. Infinite Possibilities Are Not All Possibilities}

Infinite Worlds Metaphysics definitely confuses infinite possibilities with all possibilities. "All" includes "infinite," but "infinite" does not entail "all." God or Mother Spacetime could create an infinite number of good universes without creating a corresponding number of evil universes. Plenitude of creation requires both; but plenitude as a perfection is radically different from and incompatible with the perfection of moral goodness, which adds responsible and purposive moral selectivity to the requirement to produce infinite actualities.

As the ancient Stoics and nineteenth-century Friedrich Nietzsche contended, infinite time (or space) could simply repeat an identical set of mass/ energy configurations, individuals, qualities, and relations an infinite number of times in an Eternal Recurrence of sameness. They supposed that life would participate in Eternal Recurrence, but what would guarantee this? An everlasting or an eternally recurring universe composed of a single hydrogen atom would result in no life or meaningful diversity; neither would an eternally recurring universe composed of an infinite number of hydrogen atoms (and no others). Both are logically possible universes that could eternally recur. That all universes are merely hydrogen universes is logically possible. "All" does not translate into "infinite diversity"!

Despite incessant claims to the contrary, a monkey banging on a typewriter for an infinite amount of time would not necessarily write the Bible or all the works of Shakespeare. Infinite time alone could not and would not prevent a secretarial monkey from banging on just one key-forever. Its doing so forever is logically possible, and if all possibilities are actual, it must be so! If the monkey is bored (teleology), it might strike a diversity of keys. At first, it would produce only trash. In an infinite amount of time, it would produce only an infinite amount of trash. Garbage in, garbage out-forever.

The secretarial monkey universe actually raises more questions than it answers. Who or what created the monkey? Who created the typewriter? Does only one monkey exist? Did it have parents? Does it have a navel? What does it eat? Does it ever take a break? Why does it type rather than doing something else? How does it live forever? Has its universe been around forever? How so? Some things that sound possible in the abstract are not very plausible in the concrete. The example requires a cosmology, which requires a metaphysics, which requires a theology! 
Those who assume that an infinitely diverse temporal or spatial series (which requires an Agent of Selection and Diversification) will exhaustively actualize all possibilities simply fail to understand the concept of infinity. Suppose that an infinite number of diverse individuals, qualities, and relationships were or are actualized during an infinite past or in endless space. This would not mean that all possibilities are actualized; even infinity does not use up infinity; an infinite number of universes would still remain to be actualized! Infinity subtracted from infinity still leaves infinity, and "infinite" does not mean "different" or "all."

If probability is to be our guide, without God, all past, present, and future worlds would probably be lifeless worlds. The existence of an infinite number of antecedent or contemporary universes does not explain why we live in a universe in which incomprehensibly complex and remarkable concurrences conspire to generate and support our existence and that of innumerable other inherently valuable forms of conscious life. An infinity of antecedent or coexisting universes would likely be nothing more than endless variations on inexhaustible themes of lifelessness. Life-supporting worlds are infinitely improbable, and rationality rejects infinite improbability.

\section{iv. Infinity Would Not "Use Up" Lifeless Universes}

Those who think that life-supporting universes will inevitably occur in an infinite amount of time (or space) seem to assume that certain sub-sets of infinity like lifeless universes would eventually be used up, and then a lifesupporting universe would necessarily come along; but this is not true at all. This approach treats infinity as if it were finite. It assumes that infinite sets can be used up in infinite time or space; but infinity, especially nondenumerable infinity, cannot be actualized exhaustively in any amount of space and/or time. Neither qualitative infinity, nor sub-sets of it, can be totally depleted in numerically infinite time or space. Sub-sets of infinity-all possible lifeless universescontain just as many members as infinity itself, so they can never be exhausted or used up so that something else can come along. Even after an infinite amount of time, an infinite number of lifeless universes would remain to be created. Each one could and probably would be exactly like the last unless some infinitely wise and intelligent Divine Agent of Limitation and Diversification knowingly and deliberately selects just the right conditions for life-affirming qualitative diversity.

Those who think that an infinite number of universes will actualize all possible qualitative diversity may believe that universes will be randomized, and that infinite randomization will produce infinite qualitative diversity and eventually use up all possible lifeless universes. However, randomization itself needs explaining and has its own necessary conditions. What selects and causes these conditions? Why does infinite randomization not result in infinite repetition? 
The concept of randomness makes sense only against an established background of law and order in an actually existing universe. As Alan Guth recognizes, "'randomness' is ill defined. The word 'random' does not become meaningful until the probability rules are stated. ${ }^{" 40}$ But no established finitistic probability rules exist for pure sets of all possible universes.

Given the orderly background of the actual finite laws of physics, cards in a deck can be randomly shuffled; but with no actual laws of physics, no actual cards, and no actual shufflers, no random shuffle can occur. Randomization of actualities makes no sense in a realm of undifferentiated possibilities devoid of any and all background order; and randomization alone could not use up infinity to insure that randomly produced lifeless universes are not repeated endlessly.

Given the orderly background of quantum Superspacetime and the laws of quantum physics, quantum spontaneity might generate randomness; but what guarantees the existence of Superspacetime, the ubiquity of quantum laws within it, and the exclusion of all possible non-quantum universes? Why does Superspacetime exemplify quantum instead of non-quantum laws? Alternatives are logically possible! Quantum-foamy Superspacetime cannot use up all possibilities, for a uniformly featureless Newtonian/Kantian absolute Superspacetime is logically possible also.

A teleological explanation for quantum universes is readily available and highly plausible. A creator God wanting to make free, responsible, and cocreative creatures would choose quantum laws, not Newtonian laws, for created universes in order to make room for creaturely freedom, responsibility, and creativity. God would just make spontaneously creative creatures ranging from sub-atomic particles to conscious animals and human beings. Quantum laws actually presuppose their existence, for laws are products of the average behavior of concrete entities, not efficient causes that antedate and constrain their behavior. This is also true of the laws of sociology and psychology.

\section{v. Actualizing All Possibilities Is Incoherent}

Finally, the very idea of actualizing all possibilities is logically incoherent. Possibilities are both negative and positive. Possibly, all universes are lifesupporting; possibly, none are. Possibly, the physics of every world is Newtonian; possibly the physics of every world is quantum/relativity; but these universalized possibilities cannot both be actual. Possibly the epoch that preceded ours was Newtonian, or it was open or flat; but then quantum effects in an antecedent singularity or in antecedent nothingness would not account for our world. (They don't anyway.) If all possibilities were actual, we would not be here, for it is possible that neither we nor our world should exist. Yet, we would be here, for it is possible that we and our world might exist. Possibly, all worlds are good, possibly created by a benevolent God; possibly, all worlds are bad, possibly created by a malevolent demon. A negative possibility corresponds with every 
positive possibility; and all possibilities include both! All possibilities just cancel out everything!

The Principle of Plenitude of creation is logically unintelligible. If modified to say that all "consistently combinable" possibilities are actual, who or what does the selecting? Different combinations are possible in different universes and between universes. Contradictions indicate mutually exclusive alternatives, but they do not select one or the other to be actualized. To settle this, a choice must be made. Not an abstract Principle, but an Agent of Selection and Diversification is required for this because disembodied abstract principles are impotent.

Postulating multiple universes does not account for our life-supporting universe, as it seemed to do for Schrödinger's cat that was alive in one universe but dead in another, for many possibilities that would apply to all universes are mutually exclusive. For example, it is possible that all universes are life-supporting or that none are, that all are quantum or that none are, and so on. If the "consistently combinable" qualification is accepted to begin with, Schrödinger's cat poses no problems. Unlike the Medieval theologians, Quantum Cosmologists seem to be very serious about the actualization of all possibilities-logic be damned. But logic says the same thing of Quantum Cosmologists!

\section{Infinitely Many Life-Sustaining Universes}

Suppose we knew that an infinite number of worlds exists, and all of them are good, interesting, and life-sustaining. This too is logically possible. Would this tell us anything about cosmic or supercosmic teleology? Anthropic Cosmologists themselves concede that life-supporting worlds are rare exceptions, not the rule, among possible universes. Low probabilities are possible but highly unlikely. Possibility should not be confused with probability. That each member of an infinite series of oscillating or co-existing universes handsomely supports valuable life-forms is logically possible but not very probable. Yet, if all possibilities are actual, this must be true; but it must also be false!

If a large finite or even an infinite number of universes were life-supporting and none were lifeless, what would be the most plausible explanation? Atheistic interpretations of the Anthropic Principle explain the remarkable lifesupporting coincidences of our universe by postulating an infinite number of antecedent or contemporary worlds, and by equating numerical infinity with infinite diversity. Given an infinite number of qualitatively diverse shoes, at least one will fit accidentally, they say, even though it is logically possible that none will fit, or that all will be exactly alike-all size eight when you wear a ten, or all left feet. But suppose that an infinite number of shoes all fit! No mere chance depletion of infinite diversity could explain it, for there would be no relevant diversity; no lifeless universes would be used up. Why would each 
world support life when endless lifeless worlds are logically possible? Only a Divine Agent of Limitation and Diversification could explain it.

Abstract principles are not efficient causes; they are efficacious only when embodied in and acted out by concrete decision-making individuals. Only a concrete individual agent of Divine proportions could select the right conditions for worthwhile life even once, much less an infinite number of times. If lifesupporting universes are infinitely improbable subsets of a nondenumerable infinity of possible individuals, qualities, and relations, these subsets would be actualized only if a deliberate and informed choice is made between them and infinite subsets of lifeless universes. A following chapter on the Teleological Argument for the existence of God concludes that God made the choice.

Just one life-supporting universe is infinitely improbable, and so are an infinite number of them. To be sure, we do not know that any other universes exist, much less an infinite number; but one interesting argument for the existence of an infinite number of life-supporting universes should be considered, even if it is finally rejected.

If the constants, initial conditions, and laws of nature did not break down in the final collapse of an infinite series of oscillating universes, they would presumably be the same in every successive universe. Conservation laws, along with all others, would carry over from epoch to epoch. The First Law of Thermodynamics would be in effect from one epoch to the next, and the amount of mass/energy in each cosmic epoch, no matter how many, would be identical; no mass/energy would be lost from epoch to epoch. Conservation of mass/energy would be metaphysically everlasting.

Collectively, the harmoniously integrated laws, constants, initial conditions, energy densities, and so on, of our own universe produce sufficient qualitative richness and diversity for complex living creatures to exist. If these do not break down at the end of any cosmic epochs, they would be the same in every previous and succeeding cosmic epoch. With identical laws, physical constants, quantities of energy, and initial conditions, each preceding universe would be generally life-supporting like ours (with a fly in the ointment to be explored shortly).

An infinite series of exclusively life-supporting quantum universes would not destine every cosmic epoch to be a detailed rerun of its predecessors. This might be true of deterministic Newtonian universes, but not of indeterministic Heisenberg universes. If quantum laws always obtain, each of us would not live our lives over and over again in an endless series of Eternal Recurrences. If, like ours, all previous cosmic epochs include the laws of quantum physics, then quantum indeterminacy, fluctuations, and free choices made by complex creative agents in each epoch would preclude detailed duplication. Only general life-supporting conditions could be replicated by the laws, constants, initial conditions, and quantity of mass/energy of all earlier and later relevantly similar universes. But if endless universes are as rich in life as our own, how, why, and 
by whom were life-supporting conditions chosen for all, when infinitely improbable even for one, given all possible universes from which to choose?

The Second Law of Thermodynamics is the fly in the ointment for an interminable string of life-supporting universes. If entropy or disorder increases from one cosmic epoch to another, then chaos increases from epoch to epoch. Because more chaotic, no universe could be qualitatively similar to its predecessor after all; each would have to be much less ordered than its immediate antecedent. An infinite number of earlier increases in chaos would result in pure chaos today, and too much disorder is incompatible with complex valuable life. Since our present universe is not pure chaos, either the Second Law does not carry over from epoch to epoch, or there have not been an infinite number of prior cosmic epochs, or a Divine Being intervenes periodically to bring order out of chaos, or God just created our universe out of nothing. But which is it?

\section{E. Infinite Time and the Inverse Gambler's Fallacy}

Ian Hacking brilliantly argued in 1987 that Oscillation Cosmologists like John A. Wheeler commit the Inverse Gambler's Fallacy. ${ }^{41}$ The gambler makes a logical mistake that usually costs him dearly. He comes into a game of dice and asks how many previous rolls occurred before he arrived. If many previous rolls produced no double sixes, the gambler assumes that the chances for non-twelves have been used up; and he concludes that his chance of rolling a twelve are very high; he bets heavily on twelve-and loses. The problem is that no matter how many previous rolls, the gambler's chances are exactly the same when he starts to play as on the very first roll. Once the relevant statistics about habituated entities are available, the odds do not change. The odds are the same on every roll! This is what gamblers, both cosmic and non-cosmic, do not understand.

Hacking's essential point is easier to grasp if we consider flipping a coin. With only two sides, the odds for flipping heads or tails are equally fifty/fifty. In a fluke situation, a gambler learns that tails have come up forty-nine times in a row and assumes that the odds favoring heads on the next flip are forty-nine to one; so he bets on heads-and loses. All along, the odds are just fifty/fifty. In the inverse situation, learning that heads just turned up, the gambler bets that tails appeared on the previous forty nine tosses. He bets on that-and loses.

The inverse gambler's fallacy begins with the information that an improbable double six was just rolled. From this, the gambler infers that it must have been preceded by a large number of rolls with no double sixes. If no dies were cast since he entered the room, he waits to see how the roll comes out. If it is a double six, he infers that many previous rolls must have produced different results. He then bets that many previous rolls resulted in no double sixes-and loses. In actuality, once the odds are established and known, the statistical chances of rolling a double six are just as good (or as bad) on the next as on the millionth roll. The number of rolls makes no difference to beating the odds, and 
a single current roll makes no difference in estimating the number or kind of previous rolls. $^{42}$

Applied to Anthropic Cosmology, the inverse gambler's fallacy says that "The universe has been around for ever so long, so it is not in the least surprising that it should have got into its present orderly state. Given an old enough universe, we would expect our order to arrive eventually by mere chance."13 This Humean reasoning is fallacious, says Hacking, because "There would have been no ground for believing we have an old universe, except that it explains the present order. But it does not explain the present order. So there is no ground for believing in an old universe." ${ }^{44}$

Thus, the supposition that our orderly life-supporting universe occurs late in an infinite sequence of non-life-supporting universes does nothing whatsoever to explain its existence. There is no such thing as "occurring late in" or "using up" an infinite temporal series. In an infinite series, every universe, no matter where positioned, is preceded by infinite time and an infinite number of antecedent universes. Cosmological gamblers cannot beat those odds! And if an open universe inevitably occurs after a finite or even an infinite number of oscillations, then every universe will be open, and the very notion of infinite oscillations is incoherent.

All Atheistic Anthropic Cosmologists commit the inverse gambler's fallacy when they assume that infinitely many preceding worlds would account for our life-supporting universe. Gamblers should learn that the odds against winning are the same on every throw of the dice; and cosmologists should learn that the odds against life-supporting universes are eternally the same-infinite-no matter how many antecedents. We lack absolute certainties, but rationality bets against infinite improbability. Rationality bets on a God who knows and cares.

\section{E. Infinite Space and the Inverse Gambler's Fallacy}

Does the spatial option for the Weak and Strong World-ensemble Anthropic Principle commit the inverse gambler's fallacy? Big Fizz Cosmologies affirm that if infinitely many worlds co-exist in infinite Superspace, as Carter, Linde, Barrow, Tipler, and others believe, then somewhere in that vast simultaneous infinity, some worlds would support life, not by Divine design, but just by beating the odds. Ian Hacking thinks that the infinitely many simultaneous worlds theory does not commit the Inverse Gambler's Fallacy, for time is not a factor; this theory just deduces its conclusion directly from the Principle of Plenitude. If all possible universes simultaneously co-exist, and if our ordered world is a possible universe, then our ordered world will exist. Here "Everything in this reasoning is deductive. It has nothing to do with the inverse gambler's fallacy." ${ }^{\text {ts }}$ Our life-supporting world is just deduced from the truth of the Principle of Plenitude. But plenitude is not a truth! 
Our well-founded doubts about the Principle of Plenitude are just as cogent when applied to infinitely many worlds co-existing in Superspace as to an infinite temporal succession of cosmic epochs in Supertime. Plenitude is not an empirical, scientific truth. It originated with a peculiar theological concept of Divine Perfection, and there is no other plausible rationale for it. It cannot be employed to avoid Theism. Applied to co-existing worlds, Plenitude confuses orders of infinity and is logically incoherent. The probability is infinite that lifeless worlds would occur endlessly in mindless Superspacetime. Without some intelligent and deliberately selective Divine Agent of Limitation or Diversification, infinitely many co-existing worlds would probably all be lifeless. This is possible as well as highly probable, and if possibility equals actuality, it must be so-even if it cannot be so! World-ensemble theories fail to provide an Agent of Selection and Diversification. Infinite Superspace cannot actualize all possibilities, for no matter how many contemporary lifeless universes exist, an infinite number of lifeless universes will remain to be actualized.

\section{G. Faith vs. Reason}

In discussing the relation between the Weak Anthropic Principle and worldensemble metaphysics, Fred W. Hallberg wrote:

So all those "other" universes would exist unobserved (Gale 1981, 168). The existence of our special life-enhancing universe would be the inevitable result of chance within this larger ensemble of universes. Of course, this entire supposition of an ensemble of universes is a purely speculative idea beyond any conceivable scientific determination. An equally valid alternative supposition, that our universe expresses an intention that life and consciousness be realized, also takes us beyond what could be conceivably determined by scientific experimentation. ${ }^{46}$

Hallberg thinks that very different attitudes toward the world would be appropriate, depending on which explanation of life-supporting cosmic coincidences we accept. He concludes that "Neither choice is more factual, or realistic, than the other" and that "The weak anthropic principle limits reason in a way that leaves room for faith (Kant [1787] 1958, 29). ${ }^{.47}$

Hallberg is right about the limits of science, but perhaps he restricted reason (conceived more broadly as philosophy) prematurely. Theism has more than an equally valid claim with world-ensemble Atheism on our confidence. Hallberg does not challenge the assumption that infinite number equals infinite diversity. Consequently, he does not realize that life-affirming diversity within infinitely many worlds requires an Agent of Selection and Differentiation of Divine proportions. Chapters Ten through Twelve of this book will show that belief in God is rationally warranted. Theistic faith (confidence) need not be 
blind or irrational. All rational beings, including cosmologists, should take the theistic option much more seriously.

To summarize, Atheistic Anthropic Cosmology concedes that our universe is exceptionally fine tuned for intelligent life forms; but this can be explained, its adherents claim, without appeal to God. The universe is compatible with our existence because we would not be here if it were not, says the Weak Anthropic Principle. But why is the universe compatible with our existence? The Weak and non-theistic versions of the Strong Anthropic Principle explain our good fortune by appeal to infinite worlds metaphysics. Yet, no infinite worlds metaphysics is verifiable directly or inductively. As an explanatory hypothesis, it is derived historically from an unscientific theological Principle of Plenitude, which affirms that God would be less than perfect unless he actually creates everything that he possibly could create. The assumption that an infinite number of worlds in time and/or space would actualize all possible individuals, qualities, and relations is not true, partly because a lower is not equivalent to a higher order of infinity, partly because infinite sets cannot be used up, partly because infinite sets need contain no diversity at all, and partly because the notion of actualizing all possibilities is logically incoherent. Lifeless worlds could and most likely would be repeated infinitely, just as monkeys banging typewriters would most likely produce garbage infinitely. Infinite numbers do not translate automatically into the existence of infinite diversity. Only a super-calculating life-loving God could select conditions for one or more life-supporting universes.

Infinite temporal oscillations do not improve the odds against a life-supporting universe, according to the Inverse Gambler's Fallacy. An infinity of worlds, whether successive or co-existing, is deduced from the indefensible Principle of Plenitude. An infinite plenitude of universes does not make good sense and is supported by no good reasons because abstract, disembodied, normative principles are not efficient causes, and the notion of actualizing all possibilities for all conceivable universes is logically incoherent. Atheistic Anthropic Cosmology does not successfully account for what caused our Big Bang. 